\title{
Derivatives of 2-Aminopyridines as Inhibitors of Multidrug Resistant Staphylococcus Aureus Strains
}

\author{
Iniobong E. Ante ${ }^{1}$, Sherifat A Aboaba ${ }^{1}$, Hina Siddiqui ${ }^{2}$, Muhammad A Bashir ${ }^{2}$, Muhammad I Choudhary ${ }^{2}$ \\ ${ }^{1}$ Department of Chemistry, University of Ibadan, Ibadan, Nigeria \\ ${ }^{2}$ H.E.J Research Institute of Chemistry, International Center for Chemical and Biological Sciences, University of \\ Karachi, Karachi-75270, Pakistan
}

Correspondence: Sherifat A. Aboaba, Department of Chemistry, University of Ibadan, Ibadan, Nigeria. E-mail: saboaba@gmail.com

Received: December 27, 2017 Accepted: January 26, 2018 Online Published: January 28, 2018

doi:10.5539/ijc.v10n1p153 URL: https://doi.org/10.5539/ijc.v10n1p153

\begin{abstract}
A new series of 2-aminopyridine derivatives were synthesised. N-acylation of 2-amino-3-chloro-5-(trifluoromethyl) pyridine and 2-amino-5-(trifluoromethyl) pyridine with series of acid chlorides afforded a total of fourteen (14) amide compounds. The structures of the new compounds have been established by their IR, NMR and mass spectra data. All the compounds were tested for their activity against four (4) multi-drug resistant (MDR) bacteria Staphylococcus aureus strains using microplate alamar blue assay. The MDR-Staphylococcus aureus strains employed for this study were Epidermic Methicilin Resistant Staphylococcus aureus (EMRSA-17), Methicilin Resistant Staphylococcus aureus (MRSA-252), Epidermic Methicilin Resistant Staphylococcus aureus (EMRSA-16) and Pakistani Drug resistant clinical isolate of Staphylococcus aureus (PRSA). Other bacteria strains also used include Escherichia coli (ATCC 2592), Shigella flexenari (ATCC 12022), Staphylococcus aureus (NCTC 6571) and Pseudomonas aeruginosa (NCTC 10662). The synthesised compounds exhibited very good activity against the four MDR-Staphylococcus aureus strains of which most of the compounds showed higher potencies for inhibiting the growth of the strains than vancomycin, the standard drug employed. The compounds reported here may serve as the starting point for the design and development of MDR-S.aureus inhibitors as antibacterial agents.
\end{abstract}

Keywords: N-acylation, Acid chlorides, 2, 4-difluoro-N-(5-(trifluoromethyl) pyridin-2-yl) benzamide, Staphylococcus aureus, Antibacterial

\section{Introduction}

Methicilin Resistant Staphylococcus aureus (MRSA) infections are a major public health problem producing a large number of deaths every year worldwide (NNIS, 2004). These important nosocomial and community-acquired pathogen has developed resistance to various antibiotics (Chen et al., 2010).

Pyridine nucleus plays central role in the development of different medical agents such as antimicrobial (Remuzon et al., 1992), antiviral (Tabarini et al., 2006), anticancer (Cai et al., 2003), antifungal (Patrick \& Kinsman, 1996), antioxidant (Feng \& SSNing, 2009), antidiabetic (Rajesh \& Mukul, 2007), antitubercular and analgesic (Kasabe \& Kasabe 2010). Aminopyridine and its derivatives are key intermediates for the synthesis of important pharmaceutical and agricultural products. Aminopyridine is used in the synthesis of antihistamines including methapyilene hydrochloride and pyrilamine maleate (Hettrer, 1946), synthesis of aldipem an anxiolytic (Dimsdale, 1988), preparation of the urinary tract analgesic phenazopyridine hydrochloride (Shreve, 1943), nevirapine a drug for treatment of AIDS (Merluzzi, 1990) and an antibacterial medicine, cephapirin sodium (Crast, et al., 1973).

MRSA being resistant to many antibiotics and the pyridine ring system reported as promising potential antibacterial agents, we became interested in the synthesis of 2-aminopyridine derivatives by $\mathrm{N}$-acylation which is scarce in literature giving rise to amide analogues. 2-amino-3-chloro-5-(trifluoromethyl) pyridine and 2-amino-5-(trifluoromethyl) pyridine with selected acid chlorides in water reflux with acetonitrile and triethylamine (scheme 1 and scheme 2) gave the profile of the aminopyridine derivatives studied (Table 1). 
<smiles>CCN(C)CCCCC(C)(C)Cl</smiles><smiles>[R]C(=O)Nc1ncc(C(F)(F)F)cc1Cl</smiles>

Scheme 1. Acylation of 2-amino-3-chloro-5-(trifluoromethyl) pyridine with acid chlorides<smiles></smiles><smiles>[R]C(=O)Nc1ccc(C(F)(F)F)cn1</smiles>

Scheme 2. Acylation of 2-amino-5-(trifluoromethyl) pyridine with acid chlorides

\section{Results and Discussion}

The $\mathrm{N}$-acylation of the selected aminopyridines with series of acid chlorides to give the afore mentioned amides analogues followed the $\mathrm{SN}_{2}$ mechanism pathway (scheme 3) in which the nucleophile of the amino group attacks the carbonyl of the acid chloride to afford the expected amides using the polar aprotic solvent acetonitrile which prevents solvation and excess by-products, enhanced the yields of the amides.<smiles>[R]C(Cl)(CC)CCCNCc1ncc(C(F)(F)F)cc1Cl</smiles>

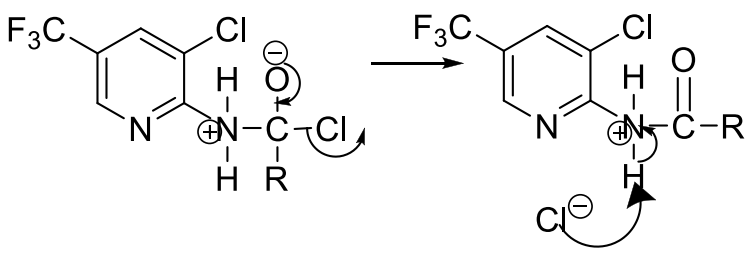<smiles>[R]C(=O)Nc1nc(C)c(C(F)(F)F)cc1Cl</smiles>

Scheme 3. General Mechanism of the synthetic reaction

Scheme 1 and scheme 2 reactions gave six (6) and eight (8) analogues which were coded as 3a-f and 5a-h respectively resulting in a total of fourteen (14) compounds. All the amides were prepared under mild conditions and the obtained products were easily purified by column chromatography on silica gel. 
http://ijc.ccsenet.org

International Journal of Chemistry

Vol. 10, No. 1; 2018

Table 1. Structural profile of the aminopyridine derivatives studied

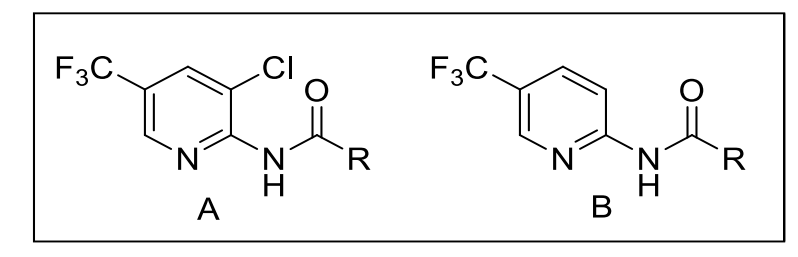

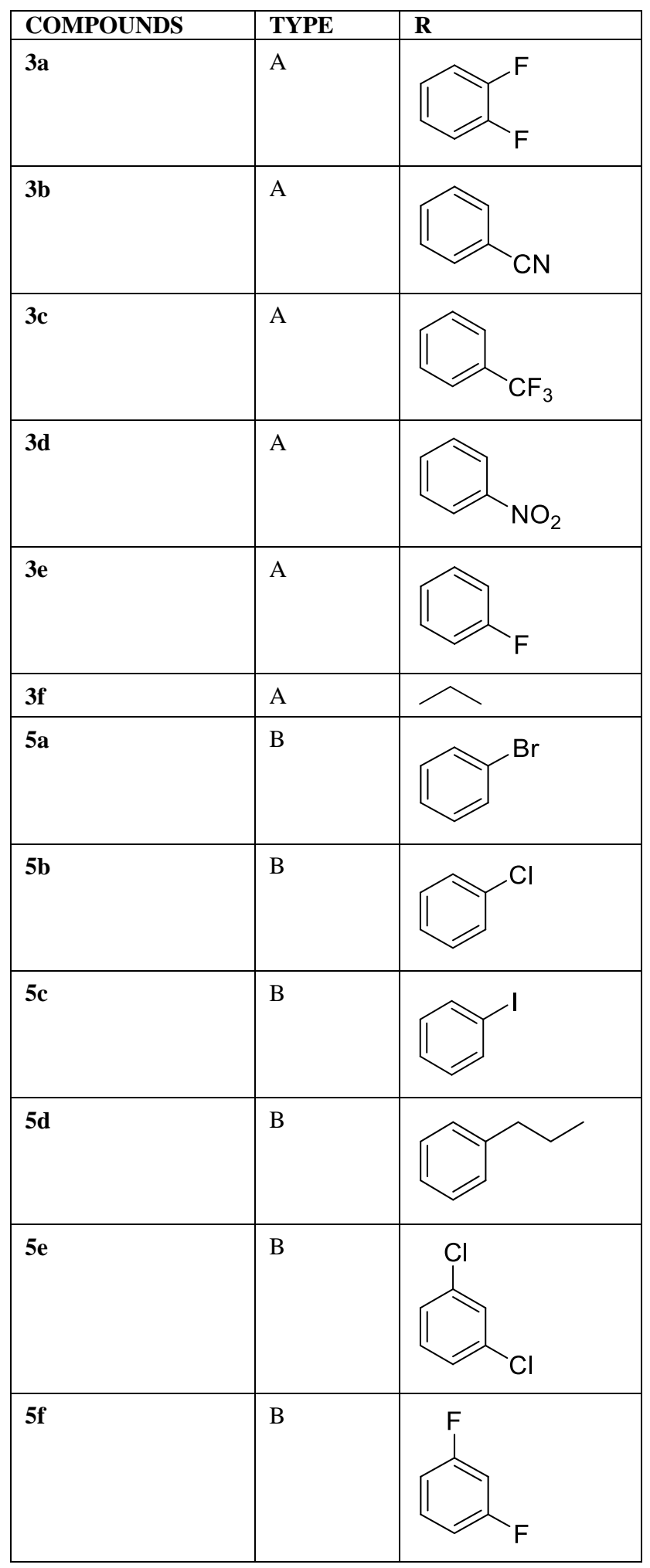

155 


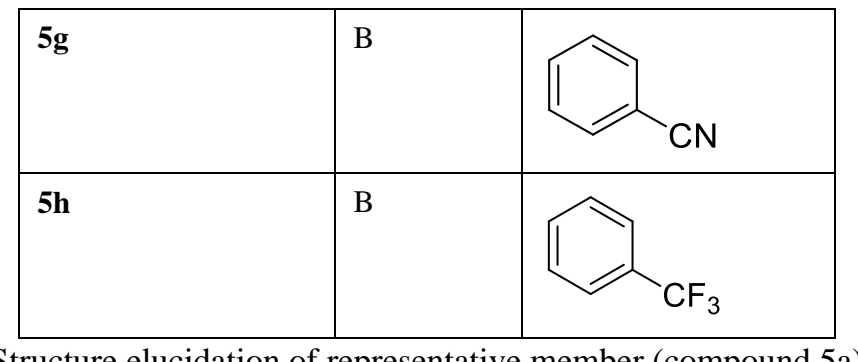

Structure elucidation of representative member (compound 5a)

The identity of the final synthesised products was determined by ${ }^{1} \mathrm{H}-\mathrm{NMR}$ and Infra Red (IR) spectroscopy and Mass Spectrometry (MS). Structure elucidation of a representative member (compound 5a) is presented below;<smiles>O=C(Nc1ccc(C(F)(F)F)cn1)c1cccc(Br)c1</smiles>

The ${ }^{1} \mathrm{H}-\mathrm{NMR}$ was performed in a $400 \mathrm{MHz}$ spectrometer and deuterated methanol MeOD was used as solvent. $\mathrm{H} 5$ was observed as a broad singlet and resonated at $\delta_{\mathrm{H}} 8.45\left(1 \mathrm{H}, \mathrm{d}, \mathrm{J}_{2,5}=9.6 \mathrm{~Hz}\right)$. The signal at $\delta_{\mathrm{H}} 8.15$ was due to $\mathrm{H} 4$ ' being coupled to $\mathrm{H}^{\prime}{ }^{\prime}\left(1 \mathrm{H}, \mathrm{d}, \mathrm{J}_{4}, 6^{\prime}=2 \mathrm{~Hz}\right)$. $\mathrm{H}^{\prime}$ ' resonated as a doublet of doublet at $\delta_{\mathrm{H}} 8.12\left(\mathrm{~J}_{6^{\prime}}{ }^{\prime}=2 \mathrm{~Hz}, \mathrm{~J}_{6}, 7_{7}=8 \mathrm{~Hz}\right)$. H2' resonated as a doublet at $\delta_{\mathrm{H}} 7.97$ being ortho coupled to $\mathrm{H}^{\prime}{ }^{\prime}\left(1 \mathrm{H}, \mathrm{d}, \mathrm{J}_{2 \cdot}{ }^{\prime}=7.6 \mathrm{~Hz}\right)$. $\mathrm{H} 3$ resonated at $\delta_{\mathrm{H}} 7.79$ as a doublet coupled to $\mathrm{H} 5\left(1 \mathrm{H}, \mathrm{d}, \mathrm{J}_{3,5}=9.6 \mathrm{~Hz}\right)$ and $\mathrm{H} 7$ ' was found coupled with both 2' and 6' and appeared as a doublet of a doublet at $\delta_{\mathrm{H}} 7.48\left(\mathrm{~J}_{7^{\prime}, 2^{\prime}}=7.6 \mathrm{~Hz}, \mathrm{~J}_{7^{\prime}, 6^{\prime}}=8 \mathrm{~Hz}\right) .{ }^{13} \mathrm{C}-\mathrm{NMR}: \delta 165.72(\mathrm{C}=\mathrm{O}), 156.03(\mathrm{C}-1), 146.3(\mathrm{C}-5), 137.07\left(\mathrm{C}-3^{\prime}\right)$, 136.5 (C-6'), 135.97 (C-3), 131.7 (C-4'), 131.4 (C-7'), 127.7 (C-2', C-6) 122.9 (C-5'), 114.6 (C-2); EI-MS m/z (rel. abund. \%): $346.8\left[\mathrm{M}^{+}+2\right]$ (2), $343.8\left[\mathrm{M}^{+}\right]$(41), 314.8 (67), 235.9 (5), 184.7 (97), 182.8 (100), 154.8 (83), 103.9 (31), 82.8 (26), 75.9 (90), 57 (77); $v_{\max }: 3433.1,3234.4,31933.9,3107.1,2923.9,2854.5,1689.5,1593.1,1240$.

The synthesised compounds were tested for their activity against four multi-Drug resistant (MDR) bacteria $S$. aureus strains and four other bacterial strains. The MDR- S. aureus strains employed for this study were Epidermic Methicilin Resistant Staphylococcus aureus (EMRSA)-17; methicilin resistant Staphylococcus aureus (MRSA)-252, Epidermic methicilin resistant Staphylococcus aureus (EMRSA)-16 and Pakistani drug resistant clinical isolate of S. aureus (PRSA) and the bacterial strains used were E.coli (ATCC 2592), S. flexanari (ATCC 12022), S.aureus (NCTC 6571) and $P$. aeruginosa (NCTC 10662) Table 2.

Table 2. Bioactivity of the compounds

\begin{tabular}{|c|c|c|c|c|c|c|c|c|c|c|c|c|c|c|c|}
\hline \multirow{2}{*}{$\begin{array}{l}\text { BACTERIAL } \\
\text { STRAINS }\end{array}$} & \multicolumn{14}{|c|}{ SYNTHESISED COMPOUNDS } & \multirow{2}{*}{$\begin{array}{l}\text { CONTROL } \\
\text { Vancomycin } \\
(20 \mu \mathrm{g} / \mathrm{ml})\end{array}$} \\
\hline & $\mathbf{3 a}$ & $\mathbf{3 b}$ & $3 c$ & 3d & $3 e$ & $3 f$ & $5 \mathbf{a}$ & $5 \mathbf{b}$ & $5 c$ & $5 d$ & $5 e$ & $\mathbf{5 f}$ & $5 g$ & $5 \mathbf{h}$ & \\
\hline EMRSA-17 & 35.9 & 36.6 & 26.8 & 28.4 & 38.9 & 39.4 & 24.5 & 13.0 & 12.1 & - & 36.1 & 41.4 & 27.4 & 43.1 & 20.7 \\
\hline MRSA-252 & 16.7 & 9.6 & 8.9 & - & 20.2 & 33.6 & - & - & - & - & 24.7 & 18.3 & 18.0 & 15.0 & 18.6 \\
\hline EMRSA-16 & 28.4 & 29.0 & 21.4 & 11.6 & 31.7 & - & - & - & - & - & 32.5 & 31.1 & 26.5 & 27.5 & 23.7 \\
\hline PRSA & 28.0 & 29.5 & 22.7 & 28.0 & 38.0 & 28.2 & 21.8 & 21.8 & - & - & 29.6 & 37.4 & 20.1 & 33.4 & 40.4 \\
\hline E.coli & - & 5.7 & 5.2 & - & - & 3.8 & 1.3 & 1.9 & - & 4.3 & 1.8 & 3.6 & 6.5 & 4.5 & $\begin{array}{l}\text { Oflaxacin } \\
(\mathbf{5 0} \boldsymbol{\mu g} / \mathbf{m l}) \\
87.7\end{array}$ \\
\hline S. flexenari & - & 15.6 & 3.7 & - & - & 8.3 & - & 3.0 & - & 0.8 & 14.9 & 15.3 & 10.0 & 5.6 & 89.9 \\
\hline S. aureus & - & 4.9 & 2.2 & - & - & 1.5 & - & - & - & - & 1.6 & - & - & - & 91.4 \\
\hline P. aureginosa & 12.1 & - & - & - & 15.0 & - & - & - & - & 1.5 & - & - & - & - & 84.4 \\
\hline
\end{tabular}


The antibacterial (EMRSA-17) inhibitory activity invitro Fig.1 afforded a two fold increase in the activity of the compounds against EMRSA-17 was observed with respect to vancomycin $(20.7 \mu \mathrm{g} / \mathrm{mL})$ the standard, when a fluorine compound was at the meta position C5' as in $\mathbf{5 f}(41.4 \mu \mathrm{g} / \mathrm{mL})$, and $\mathbf{3 e}(38.9 \mu \mathrm{g} / \mathrm{mL})$.

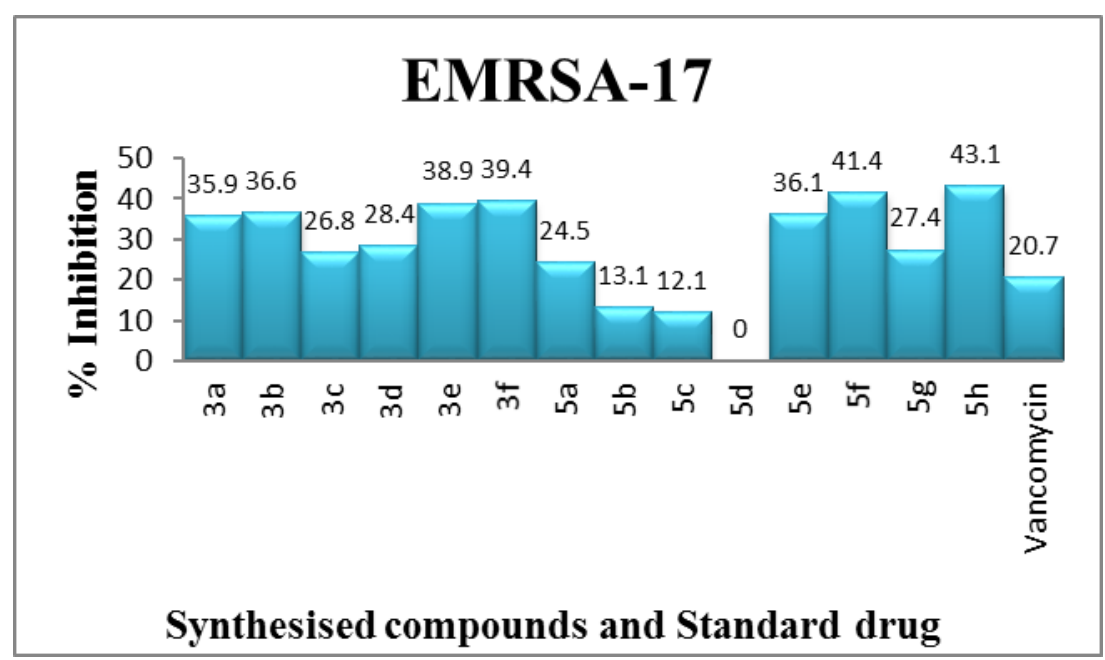

Figure 1. Inhibitory Activity against EMRSA-17

A slight decrease in activity was observed when in addition to the fluorine at the meta position another fluorine was at the ortho position C6' as observed in 3a $(35.9 \mu \mathrm{g} / \mathrm{mL})$ An electron withdrawing substituent cyano $(\mathrm{CN})$ at the para position C6' showed more increased activity when position $\mathrm{C} 2$ had chlorine as compared with $\mathbf{3 b}(36.6 \mu \mathrm{g} / \mathrm{mL})$ than when position $\mathrm{C} 2$ had no substituent as seen in $\mathbf{5 g}(27.4 \mu \mathrm{g} / \mathrm{mL})$. The absence of the benzylic ring system substituted with the presence of an electron donating alkyl chain was observed to increase the activity as seen in $\mathbf{3 f}(39.4 \mu \mathrm{g} / \mathrm{mL})$., when the end of the alkyl chain was replaced with benzene; an electron withdrawing group as against the methyl that was an electron donating group, a total loss of activity was observed as in $\mathbf{5 d}$.

The synthesised compounds were tested for their antibacterial (EMRSA-16) activity Fig.2. Significant level of activity was observed for the compounds with respect to the standard vancomycin $(23.7 \mu \mathrm{g} / \mathrm{mL})$ when the substituent at the meta position C5' was a halogen substituent fluorine as observed in $\mathbf{3 a}(28.4 \mu \mathrm{g} / \mathrm{mL}), \mathbf{3 e}(31.7 \mu \mathrm{g} / \mathrm{mL})$ and $\mathbf{5 f}(31.1 \mu \mathrm{g}$ $/ \mathrm{mL}$ ), though a total loss of activity was observed when the meta position $\mathrm{C}^{5}$ ' contained another halogen bromine and chlorine as in $\mathbf{5 a}$ and $\mathbf{5 b}$ respectively.

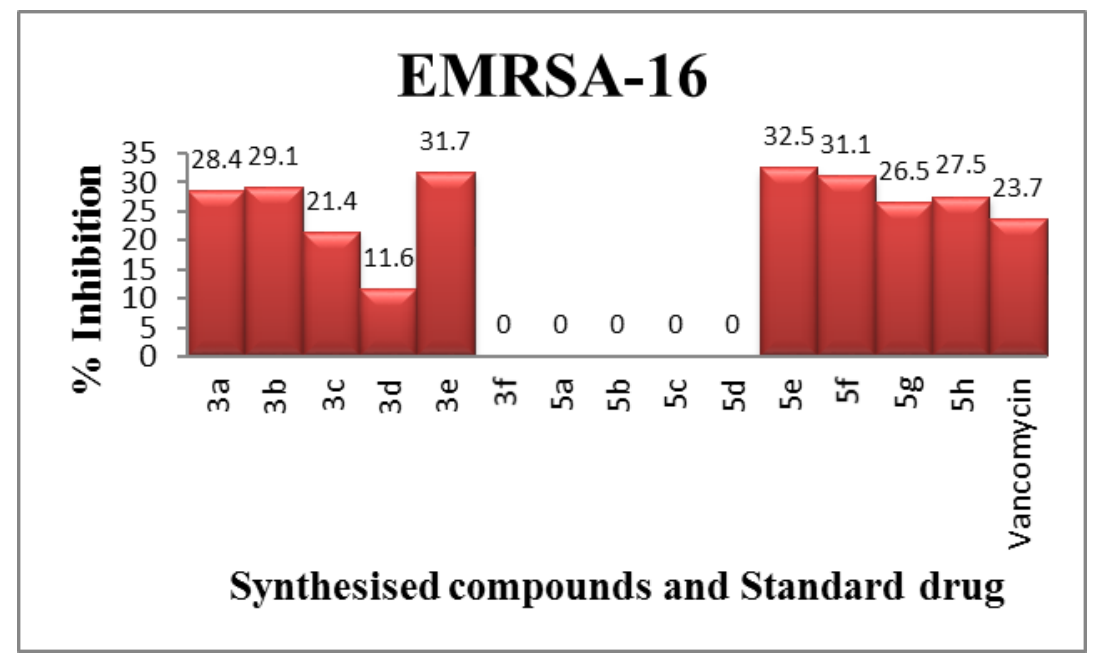

Figure 2. Inhibitory Activity against EMRSA-16

Moreso, with trifluoromethyl at position C6', significant activity was recorded similar to $\mathbf{3 c}(21.4 \mu \mathrm{g} / \mathrm{mL})$ and $\mathbf{5 h}(27.5$ $\mu \mathrm{g} / \mathrm{mL}$ ). This could have been due to the electron withdrawing ability of the halogen, fluorine present. When position C6' was substituted with yet another electron withdrawing group cyano $(\mathrm{CN})$, same trend of significant activity was observed as in $\mathbf{3 b}(29.1 \mu \mathrm{g} / \mathrm{mL})$ and $\mathbf{5 g}(26.5 \mu \mathrm{g} / \mathrm{mL})$. The highest activity against EMRSA-16 was recorded for, when chlorine was the substituent at the para position C6' as reported in $5 \mathbf{e}(32.5 \mu \mathrm{g} / \mathrm{mL})$, the second chlorine atom at the ortho position C2' might have contributed to its activity because a halogen, iodine at the C6' position resulted to a total 
loss in activity as in 5c. When there was no substituent at both meta and para positions C5' and C6', a total loss of activity was observed compared to $\mathbf{3 f}$ and $\mathbf{5 d}$.

Antibacterial (MRSA-252) inhibitory activity was performed Fig. 3. The highest activity against MRSA-252 was recorded when an alkyl chain was attached next to the carbonyl similar to $\mathbf{3 f}(33.6 \mu \mathrm{g} \mathrm{mL})$ with respect to the standard, vancomycin $(18.6 \mu \mathrm{g} / \mathrm{mL})$.

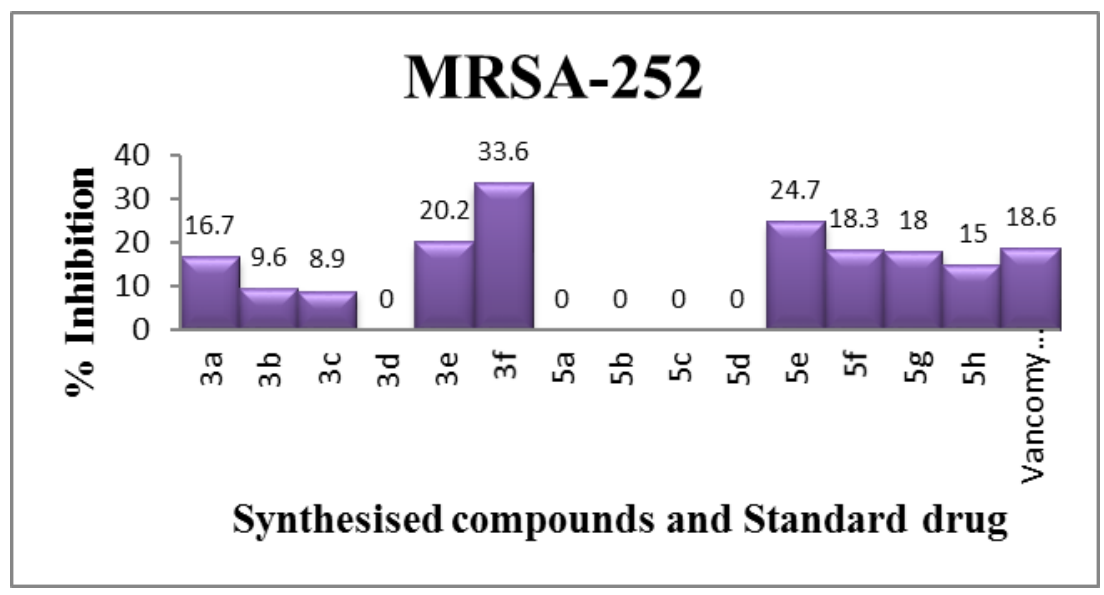

Figure 3. Inhibitory Activity against MRSA-252

The free rotation of the single bonds and the electron donating properties of the methyl and methine could have contributed. The next significant activity observed against MRSA-252 was when the para position C6' had chlorine and meta position C5' had fluorine substituents as in $\mathbf{5 e}(24.7 \mu \mathrm{g} / \mathrm{mL}), \mathbf{3 e}(20.2 \mu \mathrm{g} / \mathrm{mL}), \mathbf{5 f}(18.3 \mu \mathrm{g} / \mathrm{mL})$ and $\mathbf{3 a}(16.7 \mu \mathrm{g}$ $/ \mathrm{mL}$ ) respectively. Again, a total loss of activity was also observed for MRSA-252 strain when there was no substituent at the para position C6' as seen in $\mathbf{5 a}, \mathbf{5 b}, \mathbf{5 c}$ and $\mathbf{5 d}$ with exception of $\mathbf{5 f}$ which had fluorine at the meta position. A new trend of total loss of activity was observed for this strain of MDR S.aureus when position C6' substituent was a nitro group $\left(\mathrm{NO}_{2}\right)$ as in $\mathbf{3 d}$. This could be due to the electron donating effect of the lone pairs of electrons present in both nitrogen and oxygen; probably the mesomeric effect of both nitrogen and oxygen superseded its individual inductive effect for this particular strain. There was moderate activity observed when cyano and trifluoromethyl were at the para position C6' $(18 \mu \mathrm{g} / \mathrm{mL})$ and $\mathbf{5 h}(15 \mu \mathrm{g} / \mathrm{mL})$, a decrease in activity by half in addition to the para position still maintaining the cyano and trifluoromethyl was observed when chlorine was at position $\mathrm{C} 2$ similar to $\mathbf{3 b}(9.6 \mu \mathrm{g} / \mathrm{mL})$ and $3 \mathbf{c}(8.9 \mu \mathrm{g} / \mathrm{mL})$ respectively.

The antibacterial activity against PRSA Fig. 4 was observed to follow trend as the previous MDR-S.aureus strain afore mentioned.

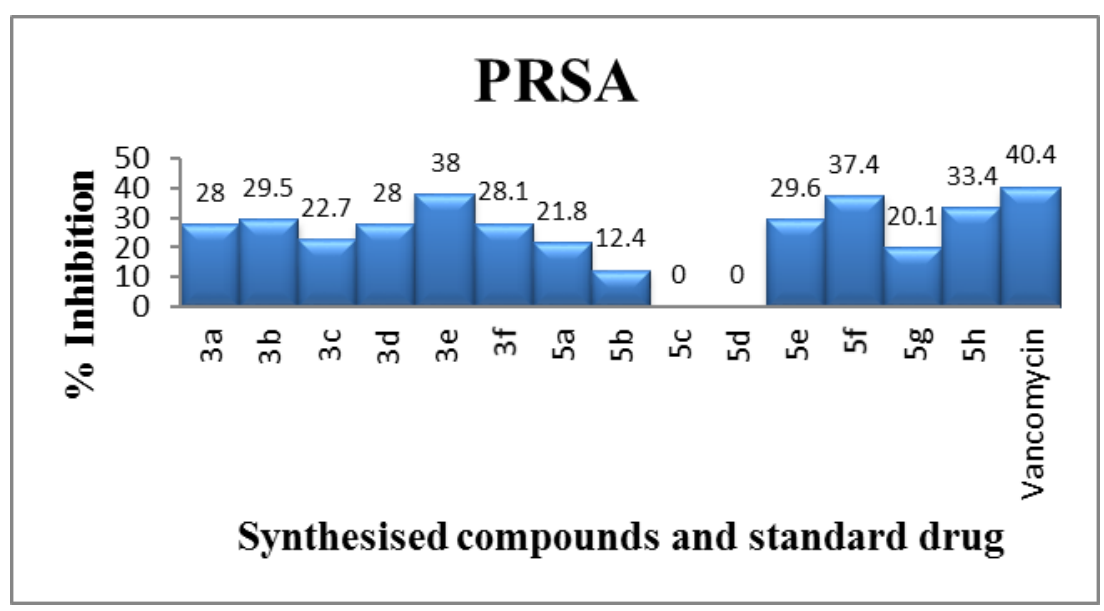

Figure 4. Inhibitory Activity against PRSA

The most significant activity with respect to the standard, vancomycin $(40.4 \mu \mathrm{g} / \mathrm{mL})$ was noticed when the meta position C5' had fluorine similar to $3 \mathbf{e}(38.0 \mu \mathrm{g} / \mathrm{mL})$ and $\mathbf{5 f}(37.4 \mu \mathrm{g} / \mathrm{mL})$ followed by when the substituent at the para position C6' was trifluoromethyl and chlorine as in $\mathbf{5 h}(33.4 \mu \mathrm{g} / \mathrm{mL})$ and $\mathbf{5 e}(29.6 \mu \mathrm{g} / \mathrm{mL})$ respectively. 


\section{Conclusion}

It could be inferred that the electron withdrawing group at the meta position C5' with reference to fluorine especially and para position C6' with reference to the cyano and trifluoromethyl have a significant role and could be the key factor for the activities observed for the synthesised compounds against these MDR-S.aureus strains.

\section{Experimental}

\subsection{General}

Chemicals were supplied by Aldrich and Sigma. NMR spectra were recorded on Avance Bruker 300, 400, 500, 600 and cryprobes FT-NMR spectrometers. Chemical shifts are reported in parts per million and were recorded in deuterated methanol, Acetone and dichloromethane accordingly with tetramethylsilane as the internal standard. NMR multiplicities are indicated by the symbols: $\mathrm{s}$ (singlet), $\mathrm{d}$ (doublet), $\mathrm{t}$ (triplet), $\mathrm{q}$ (quartet), and $\mathrm{m}$ (multiplet). IR spectra were recorded on a shimadzu 8900 FT-IR spectrometer using $\mathrm{KBr}$ discs and pellets. Mass fragmentations were recorded using MAT 312 mass spectrometer.

4.2 N-Acylation

To Acetonitrile $(10 \mathrm{ml})$, the respective aminopyridines $(1 \mathrm{mmol})$ and triethylamine $(0.101 \mathrm{ml}, 1 \mathrm{mmol})$ were added. The mixture was placed on a hot plate magnetic stirrer and stirred for 20 min under water reflux before the corresponding acid chlorides (1mmol) were added. The mixture was inspected by TLC (Hexane-EtoAc). After being stirred for a further 4-6 hrs at $40-60{ }^{\circ} \mathrm{C}$, it was concentrated to dryness. The residues were purified by silica gel gradient column chromatography (Hexane-EtoAc, 85:15) to afford the desired compounds (overall yield 65-85\%). Their identity was confirmed by NMR and IR spectroscopy and MS spectrometry.

N-(3-chloro-5-(trifluoromethyl) pyridin-2-yl)-3, 4-difluorobenzamide (3a). Yield: ( 73\%); m.p. 78-79 ${ }^{0} \mathrm{C}$; IR: 3433 $(\mathrm{N}-\mathrm{H}), 1670(\mathrm{C}=\mathrm{O}) ;{ }^{1} \mathrm{H}-\mathrm{NMR}(\mathrm{MeOD}): \delta 8.72(1 \mathrm{H}, \mathrm{brS}, \mathrm{H} 5), 8.35(1 \mathrm{H}, \mathrm{d}, \mathrm{J}=1.2 \mathrm{~Hz}, \mathrm{H} 3), 7.95\left(1 \mathrm{H}, \mathrm{d}, \mathrm{J}=2 \mathrm{~Hz}, \mathrm{H} 4{ }^{\prime}\right)$, $7.87\left(1 \mathrm{H}, \mathrm{dd}, \mathrm{J}=2,8.8 \mathrm{~Hz} \mathrm{H} 2^{\prime}\right), 7.45\left(1 \mathrm{H}, \mathrm{d}, \mathrm{J}=8.8 \mathrm{~Hz} \mathrm{H} 7^{\prime}\right) ;{ }^{13} \mathrm{C}-\mathrm{NMR}: \delta 166.32(\mathrm{C}=\mathrm{O}), 156.23(\mathrm{C}-1), 152.9\left(\mathrm{C}^{\prime} 6^{\prime}\right)$, 152.7 (C-5'), 144.9 (C-5), 137.6 (C-3), 132.09 (C-3'), 126.6 (C-2'), 127.04 (C-2), 126.02 (C-4) 122.4 (C-6), 118.97 (C-4'), 118.74 (C-7'); MS: (m/z) $336\left([\mathrm{M}+\mathrm{H}]^{+}\right), \mathrm{C}_{13} \mathrm{H}_{6} \mathrm{ClF}_{5} \mathrm{~N}_{2} \mathrm{O}(336.01)$

N-(3-chloro-5-(trifluoromethyl) pyridin-2-yl)-4-cyanobenzamide (3b). Yield: $(67 \%)$; m.p. $141-142{ }^{0} \mathrm{C}$; IR: $\mathrm{cm}^{-1}$ $3440.8(\mathrm{~N}-\mathrm{H}), 1676(\mathrm{C}=\mathrm{O}) .{ }^{1} \mathrm{H}-\mathrm{NMR}(\mathrm{MeOD}): \delta 8.73(1 \mathrm{H}, \mathrm{brS}, \mathrm{H} 5), 8.37$ (1H, brS, H3), 8.13 (2H, d, J= 8Hz, H5', H7') $7.93\left(2 \mathrm{H}, \mathrm{d}, \mathrm{J}=8 \mathrm{~Hz}, \mathrm{H} 2^{\prime}, \mathrm{H} 4^{\prime}\right)$; MS: $(\mathrm{m} / \mathrm{z}) 325\left([\mathrm{M}+\mathrm{H}]^{+}\right), \mathrm{C}_{14} \mathrm{H}_{7} \mathrm{ClF}_{3} \mathrm{~N}_{3} \mathrm{O}(325.02)$

N-(3-chloro-5-(trifluoromethyl) pyridin-2-yl) 4(trifluoromethyl) benzamide (3c). Yield: (71\%); m.p. 80-81 ${ }^{0} \mathrm{C}$; IR: $\mathrm{cm}^{-1} 3433.1(\mathrm{~N}-\mathrm{H}), 1679.9(\mathrm{C}=\mathrm{O}) .{ }^{1} \mathrm{H}-\mathrm{NMR}(\mathrm{MeOD}): \delta 8.73(1 \mathrm{H}, \mathrm{brS}, \mathrm{H} 5), 8.37(1 \mathrm{H}, \mathrm{d}, \mathrm{J}=1.5 \mathrm{~Hz}, \mathrm{H} 3), 8.17(2 \mathrm{H}, \mathrm{d}, \mathrm{J}=$ $8.4 \mathrm{~Hz}, \mathrm{H} 2^{\prime}, \mathrm{H}^{\prime}$ '), 7.87 (2H, d, J= 8.4Hz, H5', H7'); ${ }^{13} \mathrm{C}-\mathrm{NMR}: \delta 165.26(\mathrm{C}=\mathrm{O}), 153(\mathrm{C}-1), 144.66$ (C-5), 138.17 (C-3'), 137 (C-3), 130.56 (C-6'), 129.84 (C-5', C-7'), 127.71 (C-2), 126.47 (C-2', C-4'); MS: (m/z) 368 ([M+H] $\left.]^{+}\right)$, $\mathrm{C}_{14} \mathrm{H}_{7} \mathrm{ClF}_{6} \mathrm{~N}_{2} \mathrm{O}$ (368.02).

N-(3-chloro-5-(trifluoromethyl) pyridin-2-yl) 4-nitrobenzamide (3d). Yield: (67\%); m.p. 108-109 ${ }^{0} \mathrm{C}$; IR: $\mathrm{cm}^{-1} 3435$ $(\mathrm{N}-\mathrm{H}), 1685.7(\mathrm{C}=\mathrm{O}) .{ }^{1} \mathrm{H}-\mathrm{NMR}(\mathrm{MeOD}): \delta 8.75(1 \mathrm{H}, \mathrm{brS}, \mathrm{H} 5), 8.42(2 \mathrm{H}, \mathrm{d}, \mathrm{J}=8.4 \mathrm{~Hz}, \mathrm{H} 2$ ', H4'), $8.39(1 \mathrm{H}, \mathrm{d}, \mathrm{J}=$ $1.8 \mathrm{~Hz}, \mathrm{H} 3), 8.21\left(2 \mathrm{H}, \mathrm{d}, \mathrm{J}=8.4 \mathrm{~Hz}, \mathrm{H} 5^{\prime}, \mathrm{H}^{\prime}\right)$. MS: (m/z) $345\left([\mathrm{M}+\mathrm{H}]^{+}\right), \mathrm{C}_{13} \mathrm{H}_{7} \mathrm{ClF}_{3} \mathrm{~N}_{3} \mathrm{O}_{3}(345.01)$.

N-(3-chloro-5-(trifluoromethyl) pyridin-2-yl) 3-fluorobenzamide (3e). Yield: (69\%); m.p. $65-66{ }^{0} \mathrm{C}$; IR: $\mathrm{cm}^{-1} 3438.8$ $(\mathrm{N}-\mathrm{H}), 1678(\mathrm{C}=\mathrm{O}) .{ }^{1} \mathrm{H}-\mathrm{NMR}(\mathrm{MeOD}): \delta 8.73(1 \mathrm{H}, \mathrm{brS}, \mathrm{H} 5), 8.35(1 \mathrm{H}, \mathrm{brS}, \mathrm{H} 3), 7.83\left(1 \mathrm{H}, \mathrm{dd}, \mathrm{J}=1.2 \mathrm{~Hz}, 7.6 \mathrm{~Hz}, \mathrm{H} 2^{\prime}\right)$, $7.73\left(1 \mathrm{H}, \mathrm{dd}, \mathrm{J}=2 \mathrm{~Hz}, 9.6 \mathrm{~Hz}, \mathrm{H} 6\right.$ ') $7.59\left(1 \mathrm{H}, \mathrm{dd}, \mathrm{J}=7.6 \mathrm{~Hz}, 9.6 \mathrm{~Hz}, \mathrm{H} 7\right.$ ') $7.41\left(1 \mathrm{H}, \mathrm{dd}, \mathrm{J}=1.2 \mathrm{~Hz}, 2 \mathrm{~Hz} \mathrm{H} 4^{\prime}\right) ;{ }^{13} \mathrm{C}-\mathrm{NMR}: \delta$ 165.65 (C-F, C-5'), 162.5 (C=O), 153.0 (C-1), 144.9 (C-5), 137.58 (C-3), 137.0 (C-3'), 131.90 (C-7'), 126.5 (C-2), 126.03 (C-6), 125.08 (C-2'), 122.4 (C-4), 120.7 (C-4'), 115.9 (C-6'); MS: ( m/z) $318\left([\mathrm{M}+\mathrm{H}]^{+}\right), \mathrm{C}_{13} \mathrm{H}_{7} \mathrm{ClF}_{4} \mathrm{~N}_{2} \mathrm{O}$ (318.02).

N-(3-chloro-5-(trifluoromethyl) pyridin-2-yl) butyramide (3f). Yield: (74\%); m.p. 128-129 ${ }^{0} \mathrm{C}$; IR: $\mathrm{cm}^{-1} 3433.1$ $(\mathrm{N}-\mathrm{H}), 1689.5(\mathrm{C}=\mathrm{O}) ;{ }^{1} \mathrm{H}-\mathrm{NMR}(\mathrm{MeOD}): \delta 8.64(1 \mathrm{H}, \mathrm{brS}, \mathrm{H} 5), 8.27$ (1H, d, J=1.5, H3), 2.53 (2H, t, J= 7.4, H2'), 1.81 $\left(2 \mathrm{H}, \mathrm{m}, \mathrm{H} 3{ }^{\prime}\right), 1.05\left(3 \mathrm{H}, \mathrm{t}, \mathrm{J}=7.4, \mathrm{H} 4{ }^{\prime}\right) ;{ }^{13} \mathrm{C}-\mathrm{NMR}$ : $\delta 172.01(\mathrm{C}=\mathrm{O}), 152.77(\mathrm{C}-1), 144.38(\mathrm{C}-5), 136.55(\mathrm{C}-3), 124.99$ (C-2'), 123.09 (C-6), 38.0 (C-2'), 19.18 (C-3'), 13.95 (C-4'); MS: (m/z) $266\left([\mathrm{M}+\mathrm{H}]^{+}\right), \mathrm{C}_{10} \mathrm{H}_{10} \mathrm{ClF}_{3} \mathrm{~N}_{2} \mathrm{O}$ (266.04).

3-bromo-N-(5-(trifluoromethyl) pyridin-2-yl) benzamide (5a). Yield: $(80 \%)$; m.p. $82-83{ }^{\circ} \mathrm{C}$; IR: $\mathrm{cm}^{-1} 3433.1(\mathrm{~N}-\mathrm{H})$, $1689.5(\mathrm{C}=\mathrm{O}) .{ }^{1} \mathrm{H}-\mathrm{NMR}(\mathrm{MeOD}): \delta 8.66(1 \mathrm{H}, \mathrm{brS}, \mathrm{H} 5), 8.45(1 \mathrm{H}, \mathrm{d}, \mathrm{J}=8.8 \mathrm{~Hz}, \mathrm{H} 2), 8.15\left(1 \mathrm{H}, \mathrm{dd}, \mathrm{J}=1.6,2 \mathrm{~Hz}, \mathrm{H} 4^{\prime}\right)$, $8.12(1 \mathrm{H}, \mathrm{dd}, \mathrm{J}=2,8.8 \mathrm{~Hz}, \mathrm{H6}$ ') $7.97(1 \mathrm{H}, \mathrm{dd}, \mathrm{J}=1.6,7.6 \mathrm{~Hz}, \mathrm{H} 2$ '), $7.79(1 \mathrm{H}, \mathrm{d}, \mathrm{J}=8.8 \mathrm{~Hz}, \mathrm{H} 3), 7.48(1 \mathrm{H}, \mathrm{dd}, \mathrm{J}=7.6,8.8$ Hz, H7'); ${ }^{13} \mathrm{C}^{-N M R}$ : $\delta 165.72(\mathrm{C}=\mathrm{O}), 156.03$ (C-1), 146.3(C-5), 137.07 (C-3'), 136.5 (C-6'), 135.97 (C-3), 131.7 (C-4'), 131.4 (C-7'), 127.7 (C-2', C-6) 122.9 (C-5'), 114.6 (C-2); MS:( m/z) $344\left([\mathrm{M}+\mathrm{H}]^{+}\right), \mathrm{C}_{13} \mathrm{H}_{8} \mathrm{BrF}_{3} \mathrm{~N}_{2} \mathrm{O}$ (343.98).

3-Chloro-N-(5-(trifluoromethyl) pyridin-2-yl) benzamide (5b). Yield: $(65 \%)$; m.p. 132-133 ${ }^{\circ} \mathrm{C}$; IR : $\mathrm{cm}^{-1} 3434.1$ $(\mathrm{N}-\mathrm{H}), 1691.59(\mathrm{C}=\mathrm{O}) ;{ }^{1} \mathrm{H}-\mathrm{NMR}(\mathrm{MeOD}): \delta 8.00(1 \mathrm{H}, \mathrm{dd}, \mathrm{J}=1.6,2 \mathrm{~Hz}, \mathrm{H} 4$ '), $7.93(1 \mathrm{H}, \mathrm{dd}, \mathrm{J}=1.2,7.8 \mathrm{~Hz}, \mathrm{H} 3), 7.64(1 \mathrm{H}$, 
dd, J=1.6, 8.4 Hz, H6'), 7.55 (1H, t, J=8.4, 8.8 Hz, H7'), 8.66 (1H, brS, H5), 8.45 (1H, d, J= 7.8, H2), 8.12 (1H, dd, J=2, 8.8Hz, H2'); ${ }^{13} \mathrm{C}^{-N M R}: \delta 165.82(\mathrm{C}=\mathrm{O}), 156.0(\mathrm{C}-1), 146.3(\mathrm{C}-5), 136.8\left(\mathrm{C}-5^{\prime}\right), 136.5(\mathrm{C}-3), 134.9\left(\mathrm{C}^{\prime} 3^{\prime}\right), 133.02$ (C-7'), 131.2 (C-6'), 128.8 (C-4'), 127.3 (C-2'), 114.5 (C-2); MS: (m/z) 299 ([M+H] $\left.]^{+}\right), \mathrm{C}_{13} \mathrm{H}_{8} \mathrm{ClF}_{3} \mathrm{~N}_{2} \mathrm{O}(300.03)$

4-iodo-N-(5-(trifluoromethyl) pyridin-2-yl) benzamide (5c). Yield: $(60 \%)$; m.p. $85-86{ }^{0} \mathrm{C}$; IR: $\mathrm{cm}^{-1} 3400(\mathrm{~N}-\mathrm{H})$, 1656.7(C=O); ${ }^{1} \mathrm{H}-\mathrm{NMR}(\mathrm{MeOD}): \delta 8.65(1 \mathrm{H}, \mathrm{brS}, \mathrm{H} 5), 8.45(1 \mathrm{H}, \mathrm{d}, \mathrm{J}=8.8 \mathrm{~Hz}, \mathrm{H} 2), 8.12(1 \mathrm{H}, \mathrm{dd}, \mathrm{J}=2,8.8 \mathrm{~Hz}, \mathrm{H} 3), 7.93$ $\left(2 \mathrm{H}, \mathrm{dd}, \mathrm{J}=1.6,8.4 \mathrm{~Hz}, \mathrm{H} 5^{\prime}, \mathrm{H} 7^{\prime}\right), 7.74\left(2 \mathrm{H}, \mathrm{dd}, \mathrm{J}=1.6,8.4 \mathrm{~Hz}, \mathrm{H} 2^{\prime}, \mathrm{H}^{\prime}\right) ; \mathrm{MS}:(\mathrm{m} / \mathrm{z}) 392\left([\mathrm{M}+\mathrm{H}]^{+}\right), \mathrm{C}_{13} \mathrm{H}_{8} \mathrm{~F}_{3} \mathrm{IN}_{2} \mathrm{O}$ (391.96)

3-phenyl-N-(5-(trifluoromethyl) pyridin-2-yl)propanamide (5d). Yield: $(62 \%)$; m.p. $130-131{ }^{0} \mathrm{C}$; IR: $\mathrm{cm}^{-1} 3433$ $(\mathrm{N}-\mathrm{H}), 1664.5(\mathrm{~N}-\mathrm{H}) ;{ }^{1} \mathrm{H}-\mathrm{NMR}(\mathrm{MeOD}): \delta 8.55(1 \mathrm{H}, \mathrm{brS}, \mathrm{H} 5), 8.31(1 \mathrm{H}, \mathrm{d}, \mathrm{J}=8.8 \mathrm{~Hz}, \mathrm{H} 2), 8.03(1 \mathrm{H}, \mathrm{dd}, \mathrm{J}=2,8.8 \mathrm{~Hz}$, H3), 7.23 (4H, q, H5', H6',H8'H9'), 7.18 (1H,m, H7), 3.02 (2H, t, J=7.6 Hz, H2'), 2.78 (2H, t, J=7.6 Hz, H3'); MS:(m/z) : $294\left([\mathrm{M}+\mathrm{H}]^{+}\right), \mathrm{C}_{15} \mathrm{H}_{13} \mathrm{~F}_{3} \mathrm{~N}_{2} \mathrm{O}(294.10)$

2, 4-dichloro-N-(5-(trifluoromethyl) pyridin-2-yl) benzamide (5e). Yield: (85\%); m.p. $94-95{ }^{0} \mathrm{C}$; IR: $\mathrm{cm}^{-1} 3429.2$ $(\mathrm{N}-\mathrm{H}), 1722.3(\mathrm{C}=\mathrm{O}) ;{ }^{1} \mathrm{H}-\mathrm{NMR}(\mathrm{MeOD}): \delta 8.72(1 \mathrm{H}, \mathrm{brS}, \mathrm{H} 5), 8.24(1 \mathrm{H}, \mathrm{dd}, \mathrm{J}=2.4,8.4 \mathrm{~Hz}, \mathrm{H} 3), 7.82(1 \mathrm{H}, \mathrm{dd}, \mathrm{J}=2,8.4$ Hz, H5') 7.64 (2H, d, J=8.4, H2, H4'), 7.49 (1H, d, J=2.4, H7'); ${ }^{13} \mathrm{C}-\mathrm{NMR}^{\delta} 168.67$ (C=O), 155.49 (C-1), 147.07 (C-5), 137.79 (C-6'), 137.04 (C-4'), 134.42 (C-3), 133.3 (C-3'), 131.5 (C-2'), 130.75 (C-5'), 128.3 (C-7'), 124.4 (C-2); MS: $(\mathrm{m} / \mathrm{z}) 334\left([\mathrm{M}+\mathrm{H}]^{+}\right), \mathrm{C}_{15} \mathrm{H}_{13} \mathrm{~F}_{3} \mathrm{~N}_{2} \mathrm{O}(333.99)$

3, 5-difluoro-N-(5-(trifluoromethyl) pyridin-2-yl) benzamide (5f). Yield: (76\%); m.p. 126-127 ${ }^{0} \mathrm{C}$; IR: $\mathrm{cm}^{-1} 3344.3$ $(\mathrm{N}-\mathrm{H}), 1670(\mathrm{C}=\mathrm{O}) ;{ }^{1} \mathrm{H}-\mathrm{NMR}(\mathrm{MeOD}): \delta 8.66(1 \mathrm{H}$, brs, H5), $8.44(1 \mathrm{H}, \mathrm{d}, \mathrm{J}=8.8 \mathrm{~Hz}, \mathrm{H} 2), 8.13(1 \mathrm{H}, \mathrm{dd}, \mathrm{J}=2,8.8 \mathrm{~Hz}, \mathrm{H} 3)$, $7.63\left(2 \mathrm{H}, \mathrm{d}, \mathrm{J}=2.4 \mathrm{~Hz}, \mathrm{H} 2^{\prime}, \mathrm{H} 4^{\prime}\right), 7.26\left(1 \mathrm{H}, \mathrm{d}, \mathrm{J}=2.4 \mathrm{~Hz} \mathrm{H6}{ }^{\prime}\right) ;{ }^{13} \mathrm{C}-\mathrm{NMR}: \delta 164.62$ (C-5', C-7'), $162.98(\mathrm{C}=\mathrm{O}), 155.79$ (C-1), 146.30 (C-5), 138.43 (C-5'), 136.61 (C-3), 122.72 (C-6), 112.24 (C-4', C-2'), 108.4 (C-6'); MS: (m/z) 302 $\left([\mathrm{M}+\mathrm{H}]^{+}\right), \mathrm{C}_{13} \mathrm{H}_{7} \mathrm{~F}_{5} \mathrm{~N}_{2} \mathrm{O}(302.05)$.

4-cyano-N-(5-(trifluoromethyl) pyridin-2-yl) benzamide (5g). Yield: (67\%); m.p. 98-99 ${ }^{0} \mathrm{C}$; IR: $\mathrm{cm}^{-1} 3367.5(\mathrm{~N}-\mathrm{H})$, $1678.0(\mathrm{C}=\mathrm{O}) ;{ }^{1} \mathrm{H}-\mathrm{NMR}(\mathrm{MeOD}): \delta 8.67(1 \mathrm{H}, \mathrm{brs}, \mathrm{H} 5), 8.47(1 \mathrm{H}, \mathrm{d}, \mathrm{J}=8.8 \mathrm{~Hz}, \mathrm{H} 2), 8.13\left(3 \mathrm{H}, \mathrm{d}, \mathrm{J}=8.8 \mathrm{~Hz}, \mathrm{H} 3, \mathrm{H} 5{ }^{\prime}, \mathrm{H} 7^{\prime}\right)$, $7.91\left(2 \mathrm{H}, \mathrm{d}, \mathrm{J}=8.8 \mathrm{~Hz}, \mathrm{H} 2{ }^{\prime}, \mathrm{H}^{\prime}\right)$; ${ }^{13} \mathrm{C}^{3} \mathrm{NMR}: \delta 165.91(\mathrm{C}=\mathrm{O}), 155.8(\mathrm{C}-1), 146.3(\mathrm{C}-5), 138.8\left(\mathrm{C}-3{ }^{\prime}\right), 136.6(\mathrm{C}-3)$, 133.27 (C-5', C-7'), 129.69 (C-2', C-4'), 118.64 (C-8'), 116.32 (C-6'), 114.64 (C-2); MS: (m/z) 291 ([M+H] $\left.]^{+}\right)$, $\mathrm{C}_{14} \mathrm{H}_{3} \mathrm{~F}_{5} \mathrm{~N}_{3} \mathrm{O}$ (291.06).

4-(trifluoromethyl)-N-(5-(trifluoromethyl) pyridin-2-yl) benzamide (5h). Yield: (65\%); m.p. 118-119 ${ }^{0} \mathrm{C}$; IR: $\mathrm{cm}^{-1}$ $3365.3(\mathrm{~N}-\mathrm{H}), 1708.8(\mathrm{C}=\mathrm{O})$; ${ }^{1} \mathrm{H}-\mathrm{NMR}(\mathrm{MeOD}): \delta 8.67(1 \mathrm{H}, \mathrm{brs}, \mathrm{H} 5), 8.48(1 \mathrm{H}, \mathrm{d}, \mathrm{J}=8.8 \mathrm{~Hz}, \mathrm{H} 2), 8.16(2 \mathrm{H}, \mathrm{d}, \mathrm{J}=8 \mathrm{~Hz}$, H5', H7'), 7.98 (1H, d, J=8.8Hz, H3), $7.86\left(2 \mathrm{H}, \mathrm{d}, \mathrm{J}=8 \mathrm{~Hz}, \mathrm{H} 2^{\prime}, \mathrm{H} 4\right.$ '); $\mathrm{MS}:(\mathrm{m} / \mathrm{z}) 334\left([\mathrm{M}+\mathrm{H}]^{+}\right), \mathrm{C}_{14} \mathrm{H}_{8} \mathrm{~F}_{6} \mathrm{~N}_{2} \mathrm{O}(334.05)$.

\subsection{Micro-Plate Alamar Blue Assay (MABA)}

Antibacterial susceptibility testing was performed in a 96-well micro plate. Organisms were grown in Mueller-Hinton medium. Inoculums were adjusted to $0.5 \mathrm{McFarland}$ turbidity index. The stock solutions of the compounds were prepared in DMSO in a 1:1 concentration, the stock solutions were serially diluted in Muller-Hinton broth to obtain the concentration of $20 \mu \mathrm{g} / \mathrm{ml}$ and the volume of the 96 well plates were made up to $200 \mu 1.5 \times 10^{6}$ bacterial cells were added in each of the 96-well plate. The plate was then sealed and incubated at $37^{\circ} \mathrm{C}$ for $18-20 \mathrm{hrs}$, Alamar blue dye (10\%) was dispensed in each well and shaken at $80 \mathrm{RPM}$ in a shaking incubator at $37^{\circ} \mathrm{C}$ for $3-4 \mathrm{hrs}$. Oflaxacin was used as the positive control for the bacteria isolates and Vancomycin for the MDR- $S$. aureus (DMSO showed no cidal effect on the growth of the bacterial cells). All experiment was done in triplicate. Change in colour of Alamar dye from blue to pink indicated the growth of bacteria strains. Absorbance was recorded at 570 and $600 \mathrm{~nm}$ by the ELISA reader (SpectraMax M2, Molecular Devices, CA, USA).

Percentage difference in reduction of Alamar Blue between treated and control cells were calculated using the formular:

$$
\%=\frac{\left(\varepsilon_{o x}\right) \lambda_{2} A \lambda_{1}-\left(\varepsilon_{o x}\right) \lambda_{1} A \lambda_{2}}{\left(\varepsilon_{o x}\right) \lambda_{2} A^{\prime} \lambda_{1}-\left(\varepsilon_{o x}\right) \lambda_{1} A^{\prime} \lambda_{2}} \times 100
$$

Where $\left(\varepsilon_{\mathrm{ox}}\right) \lambda_{2}=$ molar extinction coefficient of Alamar blue oxidized form at $600 \mathrm{~nm}$

$\left(\varepsilon_{\mathrm{ox}}\right) \lambda_{1}=$ molar extinction coefficient of Alamar blue oxidized form at $570 \mathrm{~nm}$

A $\lambda_{1}=$ Absorbance of test wells at $570 \mathrm{~nm}$

$\mathrm{A} \lambda_{2}=$ Absorbance of test wells at $600 \mathrm{~nm}$

$\mathrm{A}^{\prime} \lambda_{1}=$ Absorbance of control well at $570 \mathrm{~nm}$

$\mathrm{A}^{\prime} \lambda_{2}=$ Absorbance of control well at $600 \mathrm{~nm}$ 


\section{Acknowledgements}

We acknowledge The World Academy of Science (TWAS) for the Award of the 2013 ICCBS-TWAS Sandwich Postgraduate Fellowship awarded to Ms. Iniobong Ante with FR number: 3240275061 and H.E.J Research Institute of Chemistry, International Center for Chemical and Biological Sciences (ICCBS), University of Karachi, Pakistan where the fellowship was tenable.

\section{References}

Cai, S. X., Nguyen B., Jia, S., Herich, J., Guastella, J. Redd, S., Tseng, B., Drewe, J., \& Kasibhatia, S. (2003). Discovery of substituted $\mathrm{N}$-phenyl nicotinamides as potent inducers of apoptosis using a cell and caspase-based high throughout screening assay. Journal of Medicinal Chemistry, 46(12), 2474-2481. https://doi.org/10.1021/jm0205200

Chen, S. Y., Wang, J. T., Chen, T. H., Lai, M. S., Chie, W. C., Chien, K. L., ... Chang, S. C. (2010). Impact of traditional hospital strain of Methicillin-Resistant Staphylococcus aureus (MRSA) and community strain of MRSA on mortality in patients with community-onset S. aureus bacteremia. Medicine. 89, 285-294. https://doi.org/10.1097/MD.0b013e3181f1851e

Crast, L. B. Jr., Graham, R. G., \& Chehey, L. C. (1973). Synthesis of Cephapirin and related cephalosporins from 7-(alpha-bromoacetomido) cephalosporanic acid. Journal of Medicinal Chemistry, 16(12), 1413-1415. https://doi.org/10.1021/jm00270a025

Dimsdale, M. (1988). Alpidem. Drugs of the future, 13,106-109. https://doi.org/10.1358/dof.1988.013.02.62368

Feng, S., \& SSNing, M. (2009). Green chemoselective synthesis of thiazolo [3, 2-a] pyridine derivatives and evaluation of their antioxidant and cytotoxic activities. Bioorganic and Medicinal Chemistry Letters, 19, 5565-5568. https://doi.org/10.1016/j.bmcl.2009.08.046

Hettrer, C. P. (1946). Heterocyclic Amines with Antihistaminic Activity. Journal of the American Chemical Society, 68(10), 1999-2002. https://doi.org/10.1021/ja01214a037

Hishmat, O. H., Abdel Galil, F. M., \& Farrag, D. S. (1990). [C] Synthesis and antimicrobial activity of new benzofuranylpyridine derivatives. Pharmazie, 45, 793-795.

Kasabe, A. J., \& Kasabe, P. J. (2010). Synthesis, antitubercular and analgesic activity evaluation of new 3-pyrazoline derivatives. International Journal of Pharmacy and Pharmaceutical Sciences, 2, 132-135.

Merluzzi, V. J., Hargrave, K. D., Labadia, M., Grozinger, K., Skoog, M., Wu, J. C., ... Adams, J. (1990). Inhibition of HIV-1 replication by a nonnucleus reverse transcriptase inhibition. Science, 250(2486), 1411-1413. https://doi.org/10.1126/science. 1701568

National Nosocomial Infections Surveillance National Nosocomial Infections Surveillance (NNIS) System Report, data summary from January 1992 through June 2004, issued October 2004. Am J Infect Control, 32, 470-485.

Patrick, G. L., \& Kinsman, O. S. (1996). Synthesis and antifungal activity of novel aza-D- homosteriods, hydroisoquinolines, pyridines and dihydrophyridines. European Journal of Medicinal Chemistry, 31(7-8), 607-613. https://doi.org/10.1016/0223-5234(96)89557-2

Rajesh, H. B., \& Mukul, R. J. (2007). Synthesis and antidiabetic activity of 2, 5-di-substututed-3-imidazol-2-yl-pyrrolo [2, 3-b] pyridines and thieno [2, 3-b] pyridines. Bioorg. and med. Chem., 15, 6782-6795. https://doi.org/10.1016/j.bmc.2007.08.005

Remuzon, P., Bouzard, D., Cesare, P. D., Dussy, C., Jacquet, J., \& Jaegly, A. (1992). Synthesis and antibacterial activity of new 5-substituted 1-cyclopropyl-6-fluoro-7-piperazinyl-1,4-dihydro-4-oxo-1,8-naphthyridine-3-carboxylic acids. Journal of Heterocyclic Chemistry, 29(4), 985-989. https://doi.org/10.1002/jhet.5570290453

Shreve, R. N., Swaney, M. W., \& Riechers, E. H. (1943). Studies in Azo dyes 1. Preparation and Bacteriostatic properties of Azo Derivatives of 2,6-Diamino pyridine. Journal of the American Chemical Society, 65(11), 2241-2243. https://doi.org/10.1021/ja01251a066

Tabarini, O., Marufroni, G., Fravolini, A., Cecchetti, V., Sabatini, S., Clercq, E., ... Neyts, J. (2006). Synthesis and AntiBVDN activity of Acridones as new potential; Antiviral Agents. Journal of Medicinal Chemistry, 49(8), 2621-2627. https://doi.org/10.1021/jm051250z

\section{Copyrights}

Copyright for this article is retained by the author(s), with first publication rights granted to the journal.

This is an open-access article distributed under the terms and conditions of the Creative Commons Attribution license (http://creativecommons.org/licenses/by/4.0/). 